\title{
Cardiac and Hepatic miRNAs Altered Expression in Rat Embryo after Bisphenol-A (BPA) Exposure and Their Histopathological Impact (In vivo Study)
}

\author{
Ahmed S. Ahmed ${ }^{1^{*}}$ \\ ${ }^{1}$ Department of Anatomy and Embryology, College of Medicine, Tanta University, Egypt.
}

Author's contribution

The sole author designed, analysed, interpreted and prepared the manuscript.

Article Information

DOI: 10.9734/ARRB/2020/v35i330202

Editor(s):

(1) Dr. Jimenez Cardoso Enedina, Hospital Infantil de Mexico Federico Gomez, Mexico.

Reviewers:

(1) Shailja Puri, India.

(2) Idorenyin Umoh, University of Uyo, Nigeria. Complete Peer review History: http://www.sdiarticle4.com/review-history/56574

Original Research Article

Received 22 February 2020

Accepted 28 April 2020

Published 18 May 2020

\begin{abstract}
Introduction: Plastic compound are widely used in modern life. Bisphenol-A is an essential component in high quality plastic containers such as bottles and food boxes. Beside its hormonal agonist and antagonist effects, Food and drug administration (FDA) had reported it is transmitted from mother to fetus through placental circulation causing mi-RNAs mutation. mi-RNAs regulate cellular morphology and functions through its essential role in genetic expression. The aim of the present study is to investigate the effect of exposure of BPA on maternal [placenta, body weight \& blood pressure] and fetal [liver, heart \& miRNAs expression] and the impact of miRNAs expression disorders on multiorgan development, namely cardiac and hepatic development.

Materials and Methods: 60 healthy pregnant females' rats were picked up since day one of gestation and divided into three groups $(n=20)$. (c) control group gained access to drinking water containing only the vehicle (tween-80) $0.1 / \mathrm{ml}$ for three weeks.(10-BPA) group, gained access to drinking water containing $10 \mathrm{mg} / \mathrm{L}$ of BPA for three weeks.(20-BPA) group, gained access to drinking water containing $20 \mathrm{mg} / \mathrm{L}$ of BPA for three weeks. Placenta, liver and heart samples were collected and histopathological changes were recorded and mi-RNAs expression was investigated. Results: Histopathological changes were noticed in BPA related groups if compared to control group. mi-RNAs expression was found in same groups with variable extent. A significant increase was noticed in body weight and blood pressure of pregnant rats.
\end{abstract}


Conclusion: Our data examined the impact of BPA exposure on different organs and their related miRNAs expression which showed a non-neglectable effect especially on the placenta of the mother and fetal heart and liver.

Keywords: Plastic; BPA; mi-RNAs; mutation; placenta; liver; heart.

\section{INTRODUCTION}

Bisphenol A (BPA) is a synthetic compound which appears as a white powder, soluble at Tween-80 solution at twenty-one degree Celsius. BPA is used in modern plastic industry with a production of a high quality plastic materials used in the manufacturing of water bottles and food storage boxes [1]. It exhibit estrogen properties but its hormonal like effect is very weak. It was reported that the effect of BPA is 2000 times less potent than estrogen [2]. It also exhibits antagonist effect to androgen [3].

Significant level of BPA was detected in the urine of plastic industry worker $[4,5]$ which indicates its mode of excretion denoting increased hazards of coronary heart diseases [6]. Epidemiological studies reported a noticeable link between exposure to BPA and cardiovascular diseases $[7,8]$. USA Food and Drug Administration (FDA) reported that BPA can be delivered from pregnant mother to its embryos through placenta [9] affecting the development of essential organs such as cardiovascular system, central nervous system and liver $[10,11]$. The multiorgan affection of the embryo was reported to occur due to disorders in glucose metabolism and insulin resistance [12].

Disorders of some organs of the embryo such as liver and heart occur, not only due to glucose metabolism, but also result from altered miRNA expression $[13,14]$ of the embryo and the placenta of the mother [15]. Over expression of miRNA-146 leads to slower placental growth [16], miRNA-192 leads to fetal liver histopathological findings [17], miRNA-127 cause maldevelopment of fetal lung [18].

MicroRNAs (miRNAs) are small RNA molecules that affect genes expression by causing modifications to histones, they are abundant in human cell and affect more than $50 \%$ of genes and lies within the gene itself [19]. More than 5000 miRNAs had been isolated [20]. As its affect gene expression, its control the normal cellular functions and any miRNAs mutation cause hereditary diseases such as skeletal or growth disorders, leukemias, hepatocellular carcinomas and various heart diseases [21].

miRNA-712 is considered a marker for blood vessels disease atherosclerosis, miRNA-296 indicates for kidney diseases and miRNA-132 regulates synaptic development and function and any mutation in this miRNA cause neurological and psychological disorders such as bipolar disorders which considered one of the most famous hereditary disorders [22].

miRNA-206 mutation may cause addiction disorders to some drugs and alcohol, while miRNA-222 disorders may encounter obesity and insulin resistance.miRNA-208 is reported to be important for cardiac muscle development and its mutation may cause heart failure [23]. Many studies had linked between different miRNAs subtypes and cardiac development like miRNAs1, 133, 208, 21and 499 [24,25].

The aim of the present study is to investigate the effect of exposure of BPA on maternal [placenta, body weight \& blood pressure] and fetal [liver, heart \& miRNAs expression] and the impact of miRNAs expression disorders on multiorgan development, namely cardiac and hepatic development.

\section{MATERIALS AND METHODS}

\subsection{Chemicals}

The following chemicals were purchased from Sigma-Aldrich company, 3050 Spruce Street, Saint Loui, Missouri, 63103, USA.

- Bisphenol-A (BPA)

- Tween-80, used as a solvent for BPA, forming a $20 \mathrm{mg} / \mathrm{L}$, it is important to mention that tween-80 is a fluid used to emulsify BPA dispersing it in water $(0.1 / \mathrm{ml})$ forming a clear yellowish solution. As it undergoes autooxidation rapidly, it should be stored at 8-degree Celsius [26].

- Paraformaldehyde, used for sample fixation

- RNA-Later puffer and miRNAs mini kit, used for miRNAs extraction 


\subsection{Animals}

70 female rats (Sprague Dawley) were used, average weight is 250 grams, housed individually with a free access to food and water, 12/12 light / dark cycle and standard temperature is kept at 25 degree Celsius. All female rats were allowed to mate with 20 male rats, and vaginal smears were collected [27]. 60 healthy pregnant females were picked up at first day of gestation (in accordance to national and institutional guidelines). This research study was approved by Research and Ethics Committee, Quality Assurance Unit, Faculty of Medicine, Tanta University, Egypt.

\subsection{Experimental Design}

60 healthy pregnant females were picked up since day one of gestation and divided into three groups $(n=20)$.

- (c) control group gained access to drinking water containing only the vehicle (tween$80) 0.1 / \mathrm{ml}$ for three weeks.

- $\quad$ (10-BPA) group, gained access to drinking water containing $10 \mathrm{mg} / \mathrm{L}$ of BPA for three weeks.

- (20-BPA) group, gained access to drinking water containing $20 \mathrm{mg} / \mathrm{L}$ of BPA for three weeks.

The average water intake was $30 \mathrm{ml} /$ day

It is very important to mention that, drinking water was changed every 8 hours to avoid autooxidation of chemicals mentioned previously.

We recorded the body weight of mother rats and their blood pressure/twice weekly at day 2, 4, 8, $10,15,17$. A final recording of the same parameters was done at day 21 .

Volume-pressure-recording-sensor was applied to the tails of mother rats to gain the non-invasive blood pressure recording.

At the end of experiment [day 21] after recording blood pressure and body weight, caesarian sections were done to all rats and the pups were collected with average 10 pups/mother.

Samples were collected as follows, mothers' placentae, pups' livers, pups' hearts.

- Placentae were fixed in paraformaldehyde, dehydrated, embedded in paraffin, sectioned at 5 um thickness and stained with hematoxylin \&eosin [28].

- $\quad$ Livers and hearts samples were divided further into three groups.

- First group: Stored in paraformaldehyde, stained with hematoxylin \&eosin

- Second group: stored in paraformaldehyde, stained with Masson Trichrome

- Third group: homogenized for miRNA extraction as per manufacturer guidelines to extract miRNAs-13, 15, 17, 21 [heart specific] and miRNA-122 [liver specific] [29].

\subsection{Statistical Analysis}

SPSS software was used for the analysis.

\section{RESULTS}

\subsection{Placenta Hematoxylin \&Eosin Stained Sections}

C-group showed normal architecture of labyrinth, basal and deciduous zones while 10-BPA group showed thrombotic vasculopathy with amnionitis and abnormal syncytiotrophoblastic changes. 20BPA group showed fused intervillous spaces with neutrophilic infiltration (Fig. 1).

\subsection{Liver Hematoxylin \&Eosin Stained Sections}

C-group showed normal histological features in the form of large hepatic cells with prominent nuclei radiating from central veins separated with blood sinusoids, while 10-BPA and 20-BPA groups showed disturbed histological features with sinusoidal congestion (Fig. 2).

\subsection{Liver Masson's Trichrome Stained Sections}

C-group showed no fibrosis, while 10-BPA and 20-BPA groups showed fibrosis of variant degrees which was more extensive in 20-BPA group if compared with 10-BPA group (Fig. 3).

\subsection{Heart Hematoxylin \&Eosin Stained Sections}

There were areas of myositis in 10-BPA and 20BPA groups if compared to C-group which showed normal histological findings (Fig. 4). 

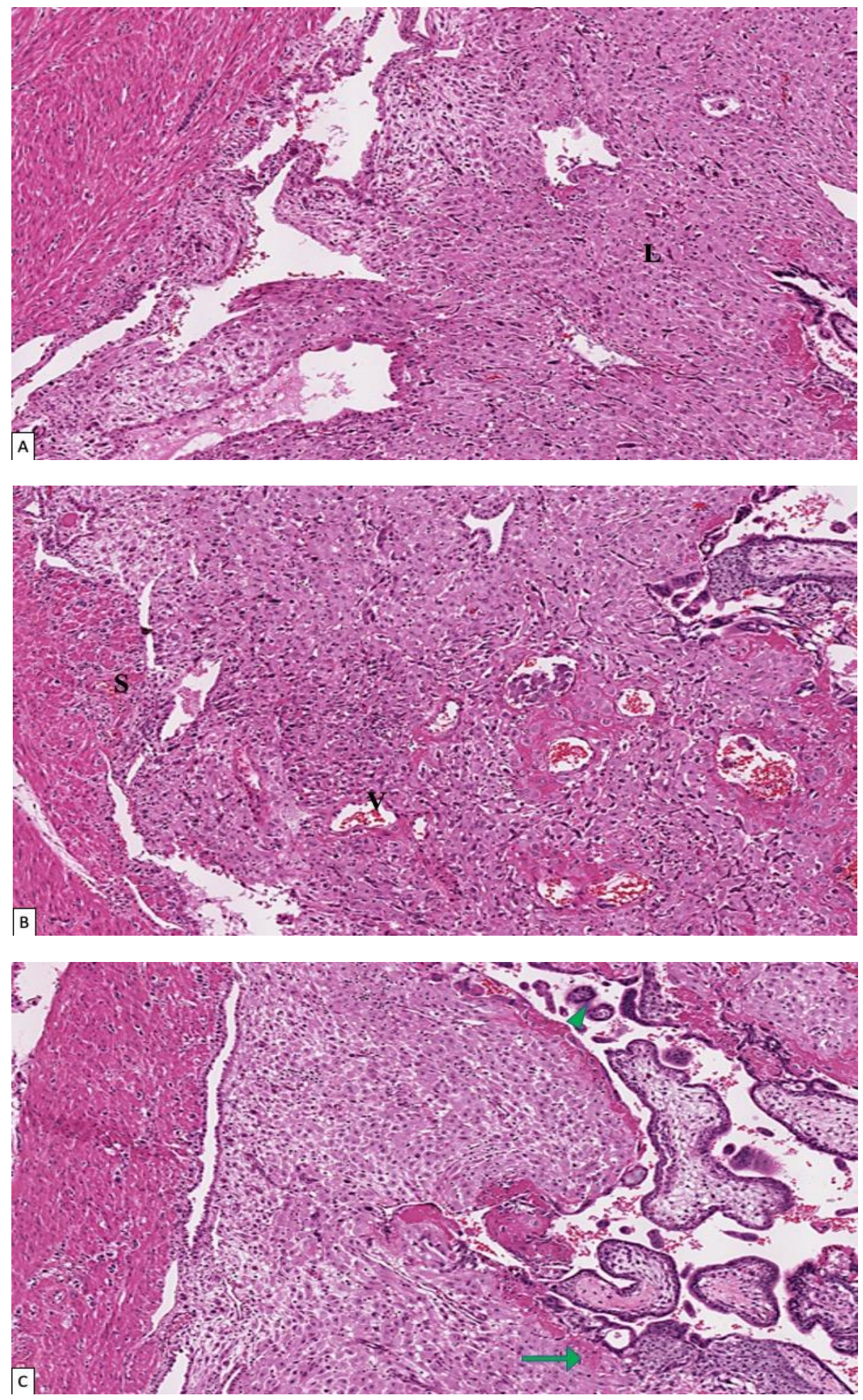

Fig. 1. A photomicrograph of placenta section stained with hematoxylin and eosin, C-group showed normal labyrinth (L) zone (A), 10-BPA-group showed thrombotic vasculopathy (V) with amnionitis and abnormal syncytiotrophoblastic (S) changes (B) and 20-BPA-group showed fused intervillous spaces (arrowhead) with neutrophilic infiltration (long arrow) (C) X1000 

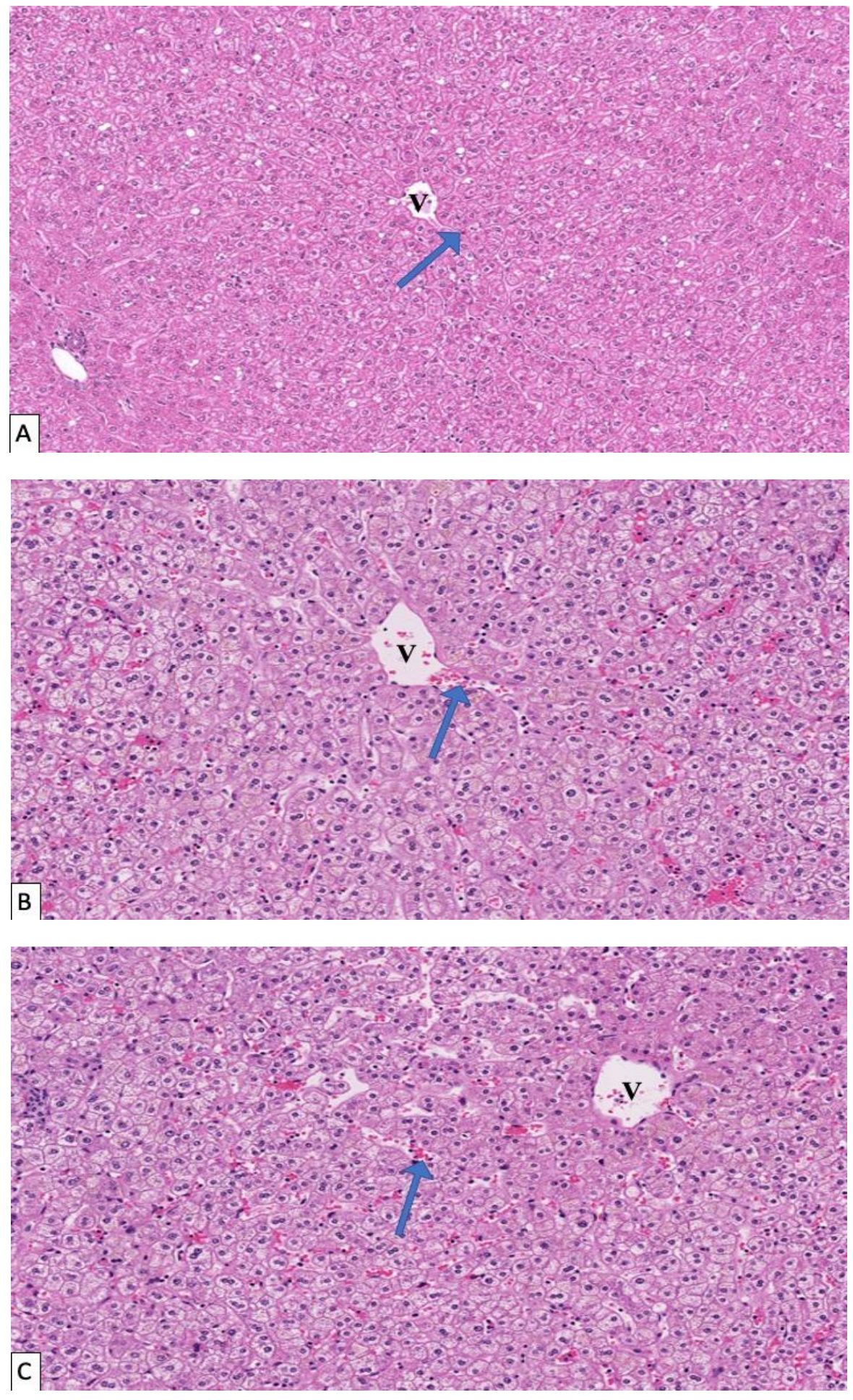

Fig. 2. A photomicrograph of liver section stained with hematoxylin and eosin, C-group showed normal histological features in the form of large hepatic cells with prominent nuclei radiating from central veins (V) separated with blood sinusoids (long arrow) (A) while 10-BPA and 20-BPA groups showed disturbed histological features with blood congested vessels (B, C) $\mathrm{X} 1000$ 

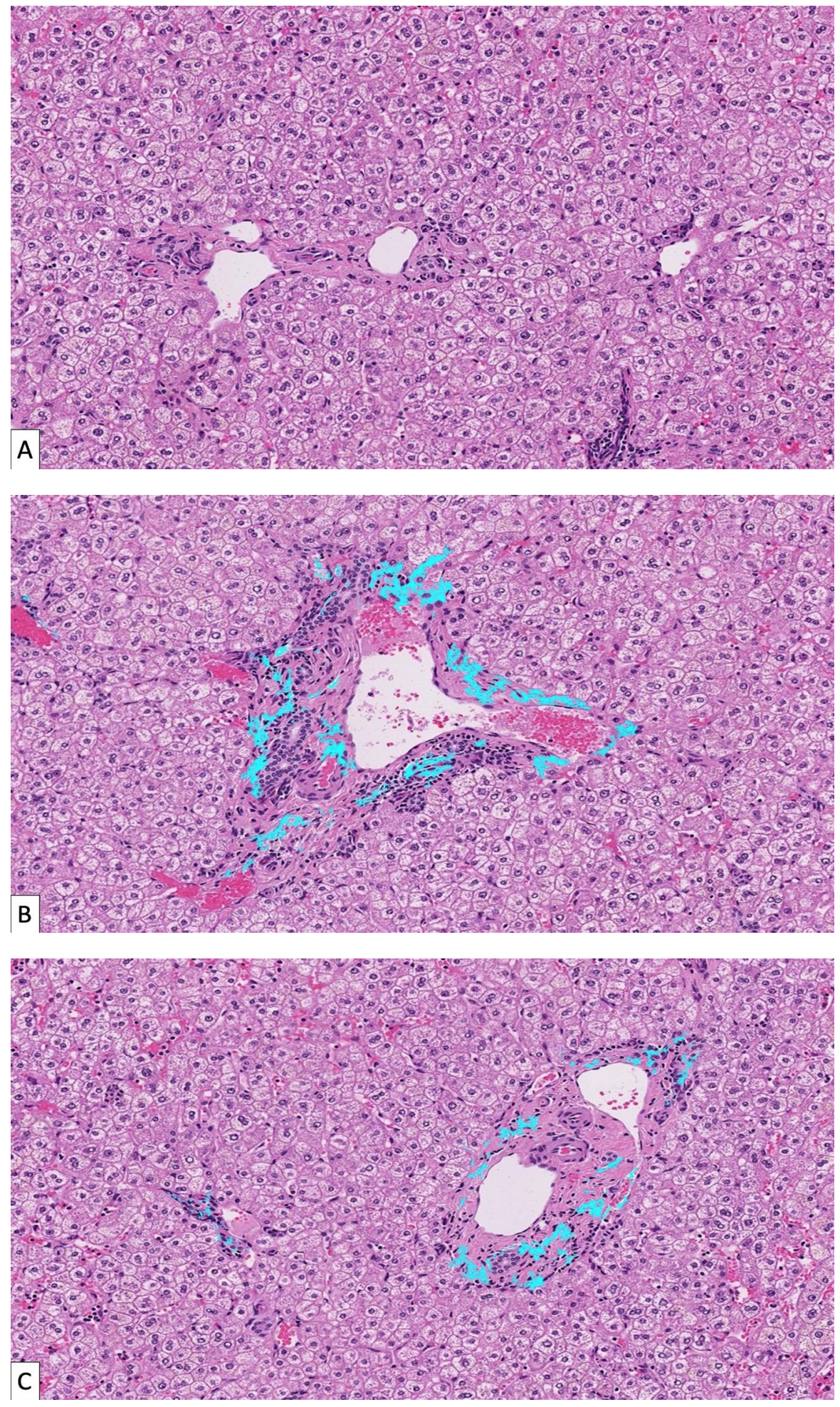

Fig. 3. A photomicrograph of liver section stained with Masson's Trichrome, C-group showed no fibrosis (A) while 10-BPA and 20-BPA groups showed fibrosis of variant degrees (B, C) $\mathrm{X} 1000$ 

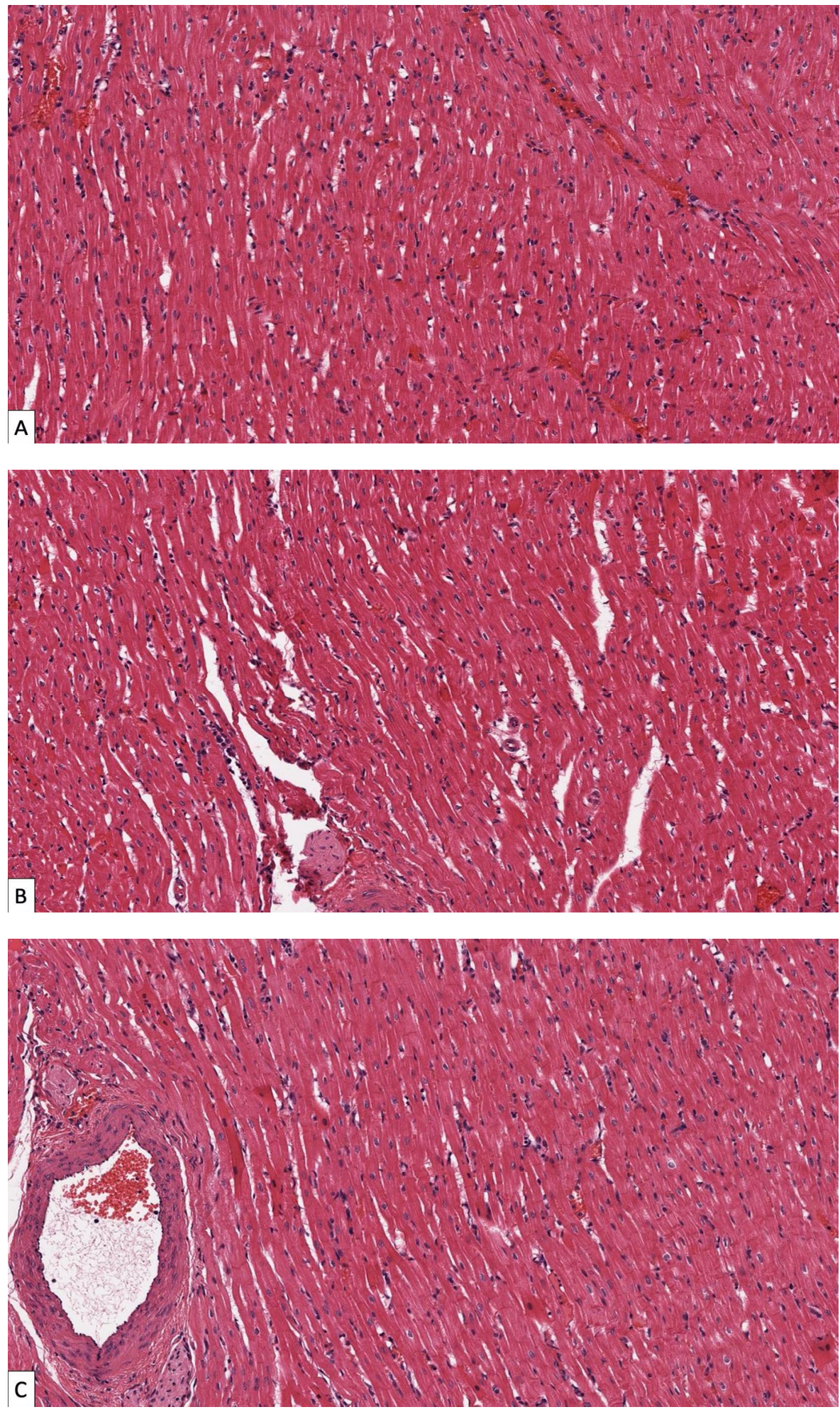

Fig. 4. A photomicrograph of heart section stained with hematoxylin and eosin, there were areas of myositis in 10-BPA and 20-BPA groups (B, C) if compared to C-group which showed normal histological findings (A) X1000 


\subsection{Heart Masson's Trichrome Stained Sections}

C-group showed no fibrosis, while 10-BPA and 20-BPA groups showed fibrosis of variant degrees which was more extensive in 20-BPA group if compared with 10-BPA group (Fig. 5).

\subsection{Body Weight}

C-group rats gained weight throughout the three weeks of pregnancy $(45 \pm 13.5 \mathrm{gm})$ while $10-\mathrm{BPA}$ and 20-BPA groups rats gained more weight which increase from $(52 \pm 20 \mathrm{gm})$ in 10-BPA group to reach $(60 \pm 13 \mathrm{gm})$ in $20-B P A$ group (Table 1).

\subsection{Blood Pressure}

C-group, third week systolic blood pressure

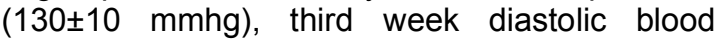
pressure $(90 \pm 10 \mathrm{mmhg})$. There was a significant increase $(P<0.005)$ of third week systolic and diastolic blood pressure in 10-BPA and 20-BPA groups if compared to C-group. As for group 10BPA systolic pressure $(137 \pm 5 \mathrm{mmhg}) \mathrm{P}=0.0052$, diastolic pressure $(100 \pm 9.5 \mathrm{mmhg}) \mathrm{P}=0.0035$.

While group 20-BPA systolic pressure (152 \pm 4.05 mmhg) $P=0.0052$, diastolic pressure $(115 \pm 3$ mmhg) $\mathrm{P}=0.0012$ (Table 2).

\section{8 mi-RNAs}

Heart specific and liver specific mi-RNAs expressions were significantly elevated in 10-BPA and 20-BPA groups, recorded as $1.6 \pm 1.3$ and $2.5 \pm 1.2$ respectively $P=0.03$ for heart specific and recorded as $1.7 \pm 1.4$ and $2.3 \pm 1.2$ respectively $\mathrm{P}=0.03$ for liver specific (Table 3).

\section{DISCUSSION}

Our study showed normal histological architecture of the placenta in control group with vasculopathy and inflammation in BPA groups this comes in agreement with [30] who reported an oxidant and inflammatory state in relation to BPA exposure. The placental affection causes a decrease in the blood supply to the developing embryo causing developmental disturbance in many organs [31].

Table 1. Mean maternal body weight during the $1^{\text {st }}, 2^{\text {nd }}$ and $3^{\text {rd }}$ weeks of gestation with significant weight increase mainly of 10-BPA and 20-BPA groups

\begin{tabular}{lllll}
\hline Week & Day & \multicolumn{3}{c}{ Weight/gm } \\
\cline { 3 - 5 } & & C-group & 10-BPA group & 20-BPA group \\
\hline $1^{\text {st }}$ & 2 & 250 & 260 & 273 \\
\multirow{2}{*}{$2^{\text {nd }}$} & 4 & 255 & 263 & 277 \\
& 8 & 266 & 275 & 280 \\
$3^{\text {rd }}$ & 10 & 270 & 282 & 293 \\
& 15 & 310 & 317 & 320 \\
& 17 & 317 & 322 & 327 \\
& 21 & 350 & 372 & 375 \\
\hline
\end{tabular}

Table 2. Mean systolic and diastolic blood pressure during the $1^{\text {st }}, 2^{\text {nd }}$ and $3^{\text {rd }}$ weeks of gestation with a significant increase in 10-BPA and 20-BPA groups

\begin{tabular}{llllllll}
\hline Week & Day & \multicolumn{6}{c}{ Blood pressure / mmhg } \\
\cline { 3 - 7 } & & \multicolumn{5}{c}{ C-group } & \multicolumn{2}{c}{ 10-BPA group } & 20-BPA group \\
\cline { 3 - 7 } & & Systolic & Diastolic & Systolic & Diastolic & Systolic & Diastolic \\
\hline $1^{\text {st }}$ & 2 & 120 & 80 & 125 & 92 & 130 & 100 \\
& 4 & 122 & 83 & 128 & 95 & 135 & 102 \\
$2^{\text {nd }}$ & 8 & 125 & 85 & 130 & 97 & 137 & 110 \\
& 10 & 127 & 87 & 132 & 98 & 140 & 113 \\
$3^{\text {rd }}$ & 15 & 130 & 90 & 137 & 100 & 152 & 115 \\
& 17 & 132 & 93 & 140 & 102 & 160 & 117 \\
& 21 & 135 & 100 & 145 & 105 & 162 & 120 \\
\hline
\end{tabular}



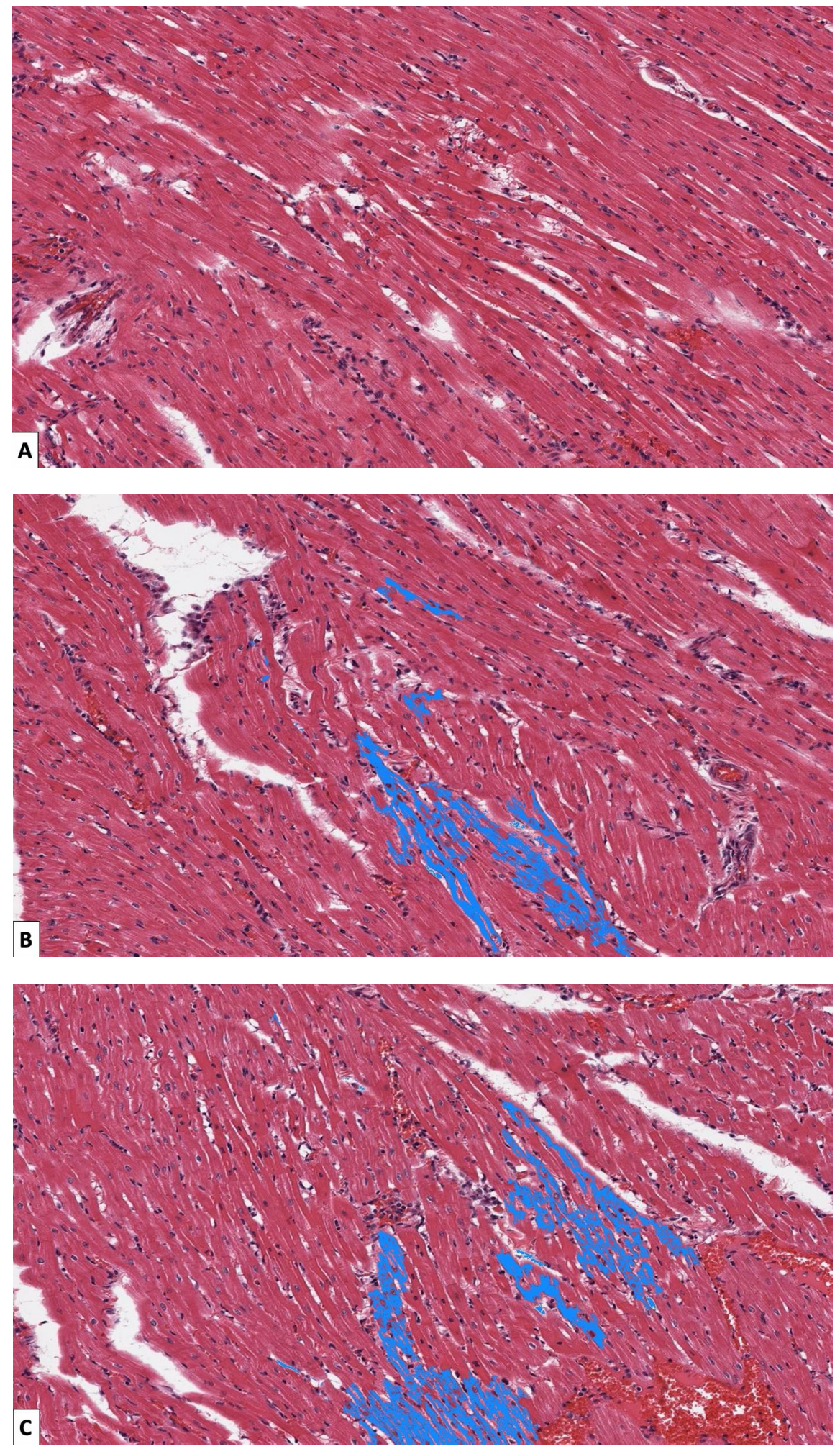

Fig. 5. A photomicrograph of heart section stained with Masson's Trichrome, C-group showed no fibrosis (A) while 10-BPA and 20-BPA groups showed fibrosis of variant degrees (B, C) $\mathrm{X} 1000$ 
Table 3. Mean heart and liver specific mi-RNAs with significant elevation of expression in 10BPA and 20-BPA groups

\begin{tabular}{llll}
\hline mi-RNA & C-group & 10-BPA group & 20-BPA group \\
\hline 13 & 1.1 & 1.2 & 1.53 \\
15 & 1 & 1.3 & 1.4 \\
17 & 1.2 & 1.5 & 1.7 \\
21 & 1 & 1.2 & 1.52 \\
122 & 1.7 & 2.1 & 2.3 \\
\hline
\end{tabular}

Our study reported histopathological changes in liver tissue in BPA related groups if compared to the control group, this was reported by another study [32] which added another dimension which is the liver functions, proven to be affected in the same study. This could be explained by miRNAs expression distortion which is reflected on the normal cellular functions. As for liver miRNA-122 expression, it may be the underlying cause of functional and structural disruption which is proved in our study after miRNA-122 extraction [33]. Histopathological changes noticed in our study in liver in BPA groups is also emphasized by the fibrosis noticed in Masson's Trichrome stained sections which comes to an agreement with $[34,35]$ who reported that exposure to BPA may cause increased expression of interleukin- 6 which acts as a fibrosis marker found in study [36] rich in collagen-I.

In our results, the muscle wall of the heart showed histopathological disturbance which comes in agreement with another study conducted on the heart tissue [37]. This suggest that BPA affects some cardiac specific miRNAs$13,15,17,21$ and any affection of their expression may affect the heart histology and function as well. Similar findings were reported in another study with higher BPA concentrations [38], this can tell that heart development and functions could be affected at lower BPA concentrations as been used in our study. The heart tissue fibrosis noticed in our study comes in the same page with [39] who reported the importance of miRNAs 133,208 in cardiomyocyte development and myosin fibers contractility. Previously, a research paper [40] stated that miRNA208 is normally absent in healthy cardiac muscle cells and any increase in its expression may cause fibrosis to heart wall.

Our study showed that there is a significant increase in the body weight of control rat which could be explained by the increased food intake, vascularity and fetal weight added. But this weight increase become more significant in BPA related groups. This comes in alignment with other studies [41]. This weight gain was explained by [42] by the adipogenesis activated by BPA. On contrary, other studies [43] reported that, there was no significant increase in weight in relation to BPA related groups if compared to control group. We explained this finding in relation to the BPA dosage used.

The significant increase in systolic and diastolic blood pressure noticed in our study especially late in pregnancy comes in agreement with study [44] who reported a significant blood pressure increase not only in the third week like our own work, but also during the second week. This finding may be due to cardiovascular system involvement as been previously shown in our results and also reported oxidant and inflammatory state reported by [45].

Many research studies had been conducted to link the gene expression modifications and the development of cardiovascular diseases [46]. Others [47] has linked the BPA exposure and the development of mal-expression of different subtypes of miRNAs. In our study we emphasized at the heard development related miRNAs especially miRNAs-13, 15, 17, 21 which showed altered expression after in vivo BPA exposure. A research paper [48] added that miRNA-21 plays a critical role in insulin formation and glucose metabolism this may be related to the obesity observed in our study in BPA related groups. Others [49], relate miRNA-21 abnormal expression with many system disorders such as respiratory system, gastrointestinal system in addition to breast carcinoma [50].

The fibrosis noticed in the heart wall in our study may be explained by the increased expression on miRNA-208, same fact was reported by various research papers [51]. Not only miRNA208 which is fibrosis related but also miRNA-17 shown in our results to have increased expression which was also reported by [52] to cause fibrosis in both cardiac and heart tissues. Distortion of expression of miRNA may affect not only the histological characteristics of the heart 
but also the cardiac function, this comes in agreement with [53] who reported heart failure in relation to BPA exposure.

\section{CONCLUSIONS}

Needless to say, that BPA exposure became a worldwide problem in modern life. BPA exposure affect the mother, maternal health and the fetal life. Our data examined the impact of BPA exposure on different organs and their related miRNAs expression which showed a nonneglectable effect especially on the placenta of the mother and fetal heart and liver. We suggest further investigations to understand the impact of BPA on a more cellular and molecular level which is affected on different organs functions later in life. Further regulation should be developed by Occupational Safety and Health Administration (OSHA) i to protect the public and plastic workers from the hazardous effect of BPA including hazardous warnings and information sharing.

\section{DISCLAIMER}

The products used for this research are commonly and predominantly use products in our area of research and country. There is absolutely no conflict of interest between the authors and producers of the products because we do not intend to use these products as an avenue for any litigation but for the advancement of knowledge. Also, the research was not funded by the producing company rather it was funded by personal efforts of the authors.

\section{COMPETING INTERESTS}

Author has declared that no competing interests exist.

\section{REFERENCES}

1. Goodson A, Robin $\mathrm{H}$, Summerfield $\mathrm{W}$, et al. Migration of bisphenol A from can coatings-effects of damage, storage conditions and heating. Food Addit Contam. 2004;121:1015-26.

2. Acconcia $F$, Pallottini $V$, Marino $M$. Molecular mechanisms of action of BPA dose response. 2015;13(4).

3. Danzl E, Sei K, Soda S, Ike M, Fujita M. Biodegradation of bisphenol $A$, bisphenol $F$ and bisphenol $S$ in seawater. Int. J. Environ. Res. Public Health. 2009;6(4): 1472-1484.
4. Bossuyt PMM, et al. Cardiovascular consequences of famine in the young. Eur. Heart J. 2011;33:538-545.

5. Van Rooij E, et al. A signature pattern of stress-responsive microRNAs that can evoke cardiac hypertrophy and heart failure. Proc. Natl. Acad. Sci. 2006;103: 18255-18260.

6. Fichtlscherer $S$, et al. Circulating microRNAs in patients with coronary artery disease. Circ. Res; 2010.

7. Cordes KR, Srivastava D. MicroRNA regulation of cardiovascular development. Circ. Res. 2009;919-928.

8. Small EM, Frost RJA, Olson EN. MicroRNAs add a new dimension to cardiovascular disease. Circulation. 2010;121:1022-1032.

9. Wang GK, et al. Circulating microRNA: A novel potential biomarker for early diagnosis of acute myocardial infarction in humans. Eur. Heart J; 2010.

10. Zhao HY, et al. The effects of bisphenol A (BPA) exposure on fat mass and serum leptin concentrations have no impact on bone mineral densities in non-obese premenopausal women. Clin. Biochem. 2012;45:1602-1606.

11. Rezg R, et al. Bisphenol A and human chronic diseases: Current evidences, possible mechanisms and future perspectives. Environ. Int. 2014;64:83-90.

12. Alonso-Magdalena $P$, et al. Bisphenol a exposure during pregnancy disrupts glucose homeostasis in mothers and adult male offspring. Environ. Health Perspect. 2010;118:1243-1250.

13. Santhi VA, Sakai N, Ahmad ED, Mustafa AM. Occurrence of bisphenol $A$ in surface water, drinking water and plasma from Malaysia with exposure assessment from consumption of drinking water. Sci. Total Environ. 2012;1428:332-338.

14. Hartle JC, Fox MA, Lawrence RS. Probabilistic modeling of school meals for potential bisphenol A (BPA) exposure. J. Expo. Sci. Environ. Epidemiol. 2016;26: 315-323.

15. Geens T, et al. A review of dietary and non-dietary exposure to bisphenol-A. Food Chem. Toxicol. 2012;50:3725-3740.

16. Christensen KLY, Lorber M. Exposure to BPA in children-media-based and biomonitoring-based approaches. Toxics. 2014;2:134-157.

17. Wang $F$, et al. High urinary bisphenol $A$ concentrations in workers and possible 
laboratory abnormalities. Occup. Environ. Med. 2012;1-7.

18. Vandenberg $L N$, Hauser $R$, Marcus $M$, Olea N, Welshons WV. Human exposure to bisphenol A (BPA). Reprod. Toxicol. 2007;24:139-177.

19. Pjanic M. The role of polycarbonate monomer bisphenol-A in insulin resistance. PeerJ. 2017;1-37.

20. Melzer D, Rice NE, Lewis C, Henley WE, Galloway TS. Association of urinary bisphenol A concentration with heart disease: Evidence from NHANES 2003 / 06. PLoS One. 2010;5:1-9.

21. Shankar A, Teppala S, Sabanayagam C. Bisphenol $A$ and peripheral arterial disease: Results from the NHANES. Environ. Health Perspect. 2012;120:12971300.

22. Rancière $F$, et al. Bisphenol $A$ and the risk of cardiometabolic disorders: A systematic review with meta- analysis of the epidemiological evidence. Environ. Heal. 2015;14-46.

23. Soriano S, et al. Rapid insulinotropic action of low doses of bisphenol-A on mouse and human islets of langerhans: Role of estrogen receptor b. PLoS One. 2012;7:19.

22. Lang $I A$, et al. Association of urinary bisphenol A concentration with medical disorders and laboratory abnormalities in adults. JAMA. 2008;300:1303-1310.

23. Domoradzki JY, et al. Age and dose dependency of the pharmacokinetics and metabolism of bisphenol $A$ in neonatal sprague- dawley rats following oral administration. Toxicol. Sci. 2004;77:230242.

24. Casas M, Forns J, Martínez D, Avellagarcía C. Exposure to bisphenol A during pregnancy and child neuropsychological development in the INMA-Sabadell cohort. Environ. Res. 2015;142:671-679.

25. Debenedictis B, Guan H, Yang K. Prenatal exposure to bisphenol A disrupts mouse fetal liver maturation in a sex-specific manner. J. Cell. Biochem. 2016;117:344350.

26. Hijazi A, Guan H, Cernea M, Yang K. Prenatal exposure to bisphenol A disrupts mouse fetal lung development. The FASEB. 2015;29:4968-4977.

27. Moustafa GG, Ahmed AAM. Impact of prenatal and postnatal exposure to bisphenol $A$ on female rats in a two generational study: Genotoxic and immunohistochemical implications. Toxicol. Reports; 2016.

28. Batista TM, et al. Short-term treatment with bisphenol-A leads to metabolic abnormalities in adult male mice. PLoS One. 2012;7:1-10.

29. Stefania Santangeli, et al. Effects of BPA on female reproductive function: The involvement of epigenetic mechanism. Gen. Comp. Endocrinol. 2017;245:122126.

30. Lin $\mathrm{Y}$, et al. Downregulation of miR-192 causes hepatic steatosis and lipid accumulation by inducing SREBF1: Novel mechanism for bisphenol A-triggered nonalcoholic fatty liver disease. Mol. Cell Biol. Lipids. 2017;1862:869-882.

31. Chapalamadugu KC, Vandevoort CA, Settles ML, Robison BD, Murdoch GK. Maternal bisphenol A exposure impacts the fetal heart transcriptome. PLoS One. 2014;9:1-9.

32. Avissar-Whiting $M$, et al. Bisphenol $A$ exposure leads to specific microRNA alterations in placental cells. Reprod. Toxicol. 2010;29:401-406.

33. Bhaskaran $M$, et al. MicroRNA-127 modulates fetal lung development. Physiol Genomics. 2009;37:268-278.

34. Heykants M, Mahabir E. Estrous cycle staging before mating led to increased efficiency in the production of pseudopregnant recipients without negatively affecting embryo transfer in mice. Theriogenology. 2016;85:813-821.

35. Zhang $\mathrm{H}$, Yao M, Morrison RA, Chong S. Commonly used surfactant, Tween 80, improves absorption of P-glycoprotein substrate, digoxin in rats. Arch. Pharm. Res. 200326:768-772.

36. Miller KP, Borgeest C, Greenfeld C, Tomic $D$, Flaws JA. In utero effects of chemicals on reproductive tissues in females. Toxicol. Appl. Pharmacol. 2004;198:111-131.

37. Wang $\mathrm{H}$, et al. Cardiomyocyte-specific deletion of the $G$ protein-coupled estrogen receptor (GPER) leads to left ventricular dysfunction and adverse remodeling: $A$ sex-specific gene profiling analysis. Biochim. Biophys. Acta. 2017;1863:18701882.

38. Gadonski G, et al. Hypertension produced by reductions in uterine perfusion in the pregnant rat. Hypertension. 2006;48:711716.

39. Wei J, et al. Perinatal exposure to bisphenol A exacerbates nonalcoholic 
steatohepatitis-like phenotype in male rat offspring fed on a high-fat diet. J. Endocrinol. 2014;222:313-325.

40. Hernández-Ramos ÁD, et al. Acanthocytosis and brain damage in area postrema and choroid plexus: Description of novel signs of Loxosceles apachea envenomation in rats. PLoS One. 2019;14:e0211689.

41. Houlihan DD, et al. Isolation of mouse mesenchymal stem cells on the basis of expression of Sca-1 and PDGFR- $\alpha$. Nat. Protoc. 2012;7:2103-2111.

42. Gray C, Li M, Patel R, Reynolds CM, Vickers $\mathrm{MH}$. Let-7 miRNA profiles are associated with the reversal of left ventricular hypertrophy and hypertension in adult male offspring from mothers undernourished during pregnancy after preweaning growth hormone treatment. Endocrinology. 2014;155:4808-4817.

43. Turchinovich A, Weiz L, Langheinz A, Burwinkel B. Characterization of extracellular circulating microRNA. Nucleic Acids Res. 2011;39:7223-7233.

44. Masè $M$, et al. Selection of reference genes is critical for miRNA expression analysis in human cardiac tissue. A focus on atrial fibrillation. Nat. Publ. Gr; 2017.

45. Tian J, An X, Niu L. Role of microRNAs in cardiac development and disease (Review). Exp. Ther. Med. 2017;13:3-8.
46. Rasdi Z, et al. Effects of bisphenol $A$ on neonatal cardiomyocytes beating rate and morphology. J. Teknol. 2018;6:141-147.

47. Xia W, et al. Early-life exposure to bisphenol $A$ induces liver injury in rats involvement of mitochondria-mediated apoptosis. PLoS One. 2014;9:1-9.

48. Zhang $Z$, et al. Urinary bisphenol A concentrations and their implications for human exposure in several Asian countries. Environ. Sci. Technol; 2011.

49. Keri RA, et al. An evaluation of evidence for the carcinogenic activity of bisphenol $A$. Reprod. Technol. 2007;24:240-252.

50. Lemmen JG, Arends RJ, van der Saag PT, van der Burg B. In vivo imaging of activated estrogen receptors in utero by estrogens and bisphenol A. Environ. Health Perspect. 2004;112:1544-1549.

51. Murray TJ, Maffini MV, Ucci AA, Sonnenschein $C$, Soto AM. Induction of mammary gland ductal hyperplasias and carcinoma in situ following fetal bisphenol A exposure. Reprod. Toxicol; 2007.

52. Nwachukwu D, Adegunloye BJ, Bello Ol. Blood pressure and heart rate changes during pregnancy in fructose-fed spraguedawley. Afr. J. Med. Sci. 2001;30:187-190.

53. Han C, Hong $\mathrm{Y}$. Bisphenol A, hypertension, and cardiovascular diseases: Epidemiological, laboratory and clinical trial evidence. Curr Hypertens Rep. 2016;11:1-5.

(c) 2020 Ahmed; This is an Open Access article distributed under the terms of the Creative Commons Attribution License (http://creativecommons.org/licenses/by/4.0), which permits unrestricted use, distribution, and reproduction in any medium, provided the original work is properly cited.

Peer-review history:

The peer review history for this paper can be accessed here: http://www.sdiarticle4.com/review-history/56574 